from work organisation and work environment factors, including irregular employment, multiple job sites, long commutes, long work hours, and employer policies regarding health and safety. These non-traditional hazards are associated with injury and illness, as well as health behaviours including poor diet, smoking, and psychosocial stress. The cumulative impact of both traditional and non-traditional hazards on the health and well-being of construction workers are largely unknown.

Methods We conducted a survey among a cohort of apprentice construction workers to identify relationships between work organisation, environmental factors, health behaviours, and health outcomes.

Results 960 surveys have been completed to date. Preliminary analyses show that respondents (mean age 28) report high levels of job satisfaction, job security, and social support, but also report high rates of musculoskeletal symptoms and various work organisational factors potentially affecting health and health behaviours. Average commuting distance to work is over $60 \mathrm{~km}$, and $63 \%$ report no limits on daily working hours. Despite high smoking rates (28\% are current smokers), only 55\% report any restrictions on smoking at their worksites. Only $10 \%$ report regularly using sunscreen when in the sun for >15 min; only 4\% of worksites provided sunscreen.

Discussion These preliminary results highlight non-traditional worksite health risks, and suggest potential interventions that may reduce the high rates of risky heath behaviours among construction workers. As our study progresses, we plan to evaluate the impact of work organisation on health and health behaviours in three construction trades; identify workplace programs, policies, and practices affecting worker health and well-being; and determine readiness for adoption of integrated interventions to improve worker health.

\section{WORKPLACE PRACTICES AND POLICIES TO SUPPORT WORKERS WITH DEPRESSION}

D Van Eerd*, K Cullen, E Irvin, MLe Pouésard. Institute for Work and Health, Toronto, Canada

\subsection{6/oemed-2018-ICOHabstracts. 1660}

Introduction The burden associated with the effects of depression in the workplace is extensive. Workers with depression lose more health-related productive time, have higher rates of absenteeism and short-term disability, and experience higher rates of job turnover than those without depression. Our objective is to synthesise evidence from the scientific literature, practice evidence (workplace policies and practices), and experiences from OHS stakeholders.

Methods Our study sample is OHS stakeholders: workers, managers, and consultants in workplaces from our contact database (approximately 700 OHS contacts across Canada willing to be contacted for research). Data collection includes a web-based survey, focus groups (ongoing), and interviews (ongoing) with stakeholder representatives from multiple sectors. We are collecting information about workplace practices and policies to prevent productivity losses, promote stay-atwork, and support return-to-work for workers with depression. The synthesis is a two stage process: first synthesising practice evidence gathered from stakeholders and then combining that with evidence from the scientific literature.

Results Preliminary results ( $n=400,73 \%$ workers, 27\% managers/consultants) reveal a willingness among participants to share their experiences with depression and work. Workers report practices related to non-judgemental listening and external supports were most helpful to them. Managers/consultants suggest non-judgemental listening and employee assistance programs were most helpful. However, workers often feel workplace support is lacking and report non-supportive supervisors as a key barrier to receiving needed support. Managers indicate a lack of training and knowledge about depression are the main barriers to providing support to workers. Workers did not feel providing information was helpful whereas managers often did.

Conclusion Preliminary results reveal the importance of nonjudgemental listening as a workplace support for depression. However, responses reveal workers and managers do not have similar experiences of workplace support. Synthesising practice and scientific evidence will help guide policies and practices to support workers with depression.

\section{PREVENTING WORK DISABILITY IN WORKERS WITH DEPRESSION; A SYSTEMATIC REVIEW}

K Cullen* , E Inin, D Van Eerd, R Saunders. Institute for Work and Health, Toronto, Canada

\subsection{6/oemed-2018-ICOHabstracts. 1661}

Introduction By the year 2020, depression will be the second most burdensome illness in developed economies. In addition to its adverse individual effects, the associated workplace effects of depression are extensive. This review is provides a synthesis of the evidence to determine effective intervention approaches for managing depression in the workplace: both to help workers stay-at-work while experiencing symptoms and to return-to-work after an episode of time away from work.

Methods We followed a systematic review process developed by the Institute for Work and Health and an adapted best evidence synthesis. Articles that met the following criteria were considered: working age individuals with depression; workplace-based interventions; including a comparison group; outcomes of work functioning, work disability, or recurrences of work disability.

Result Seven electronic databases were searched from inception up to June 2015. The review examined 8123 titles and abstracts for relevance and found 20 RCTs and seven nRCTs from various jurisdictions. These studies evaluated a range of interventions, including; cognitive-behavioural therapy (CBT), work-focused CBT, problem solving therapy (PST), workfocused PST, enhanced care delivery, coordination of services, short- and long-term psychodynamic therapy, stress reduction programs, exercise training, part-time sick leave and naturebased rehabilitation. Our findings indicate that CBT and PST interventions can help workers with depression stay-at-work while managing their symptoms; however, only work-focused CBT is sufficient to help workers return-to-work after an absence. There is currently not enough evidence from the scientific literature to guide practice for the remaining interventions identified in this review.

Discussion We synthesised the current best evidence on workplace interventions to help employees manage symptoms of depression. The interventions identified in this review focused on strategies targeting personal resilience and coping skills of individuals with depression. More work is needed to evaluate interventions aimed at mitigating workplace factors such as psychosocial working conditions. 